\title{
Prdm16 determines the thermogenic program of subcutaneous white adipose tissue in mice
}

\author{
Patrick Seale, ${ }^{1}$ Heather M. Conroe, ${ }^{2}$ Jennifer Estall, ${ }^{2}$ Shingo Kajimura, ${ }^{2}$ Andrea Frontini, ${ }^{3}$ \\ Jeff Ishibashi, ${ }^{1}$ Paul Cohen, ${ }^{2}$ Saverio Cinti, ${ }^{3}$ and Bruce M. Spiegelman' ${ }^{2}$
}

\begin{abstract}
1/nstitute for Diabetes, Obesity, and Metabolism and Department of Cell and Developmental Biology, University of Pennsylvania School of Medicine, Philadelphia, Pennsylvania, USA. '2Dana-Farber Cancer Institute and Department of Cell Biology, Harvard Medical School, Boston, Massachusetts, USA ${ }^{3}$ Department of Molecular Pathology and Innovative Therapies, School of Medicine, University of Ancona (Politecnica delle Marche), Ancona, Italy.
\end{abstract}

\begin{abstract}
The white adipose organ is composed of both subcutaneous and several intra-abdominal depots. Excess abdominal adiposity is a major risk factor for metabolic disease in rodents and humans, while expansion of subcutaneous fat does not carry the same risks. Brown adipose produces heat as a defense against hypothermia and obesity, and the appearance of brown-like adipocytes within white adipose tissue depots is associated with improved metabolic phenotypes. Thus, understanding the differences in cell biology and function of these different adipose cell types and depots may be critical to the development of new therapies for metabolic disease. Here, we found that Prdm16, a brown adipose determination factor, is selectively expressed in subcutaneous white adipocytes relative to other white fat depots in mice. Transgenic expression of Prdm16 in fat tissue robustly induced the development of brown-like adipocytes in subcutaneous, but not epididymal, adipose depots. Prdm 16 transgenic mice displayed increased energy expenditure, limited weight gain, and improved glucose tolerance in response to a high-fat diet. shRNA-mediated depletion of Prdm16 in isolated subcutaneous adipocytes caused a sharp decrease in the expression of thermogenic genes and a reduction in uncoupled cellular respiration. Finally, Prdm16 haploinsufficiency reduced the brown fat phenotype in white adipose tissue stimulated by $\beta$-adrenergic agonists. These results demonstrate that $\operatorname{Prdm} 16$ is a cell-autonomous determinant of a brown fat-like gene program and thermogenesis in subcutaneous adipose tissues.
\end{abstract}

\section{Introduction}

The rise in the incidence of obesity has driven a public health crisis because excess adiposity predisposes to cardiovascular disease, type 2 diabetes, hypertension, stroke, and many cancers $(1,2)$. Since weight gain is almost always caused by chronic energy imbalance, nonsurgical therapy for obesity must reduce energy intake and/or increase energy expenditure.

There are 2 major types of adipose tissues in mammals, white and brown. White adipose tissue (WAT) is highly adapted to store excess energy in the form of triglycerides. Conversely, brown adipose tissue (BAT) oxidizes chemical energy to produce heat as a defense against hypothermia and obesity. WAT develops in distinct intra-abdominal depots and in the subcutaneous layer (between the fascia and muscle). The accumulation of intra-abdominal, visceral WAT, rather than total adiposity, is most strongly correlated with elevated risk for metabolic dysfunction and cardiovascular disease (3-8). By contrast, expansion of subcutaneous WAT, even in the setting of obesity, has been suggested to promote insulin sensitivity in rodents and humans (9-14). Implantation of subcutaneous WAT, but not visceral WAT, into the abdominal cavity of mice improves whole-body metabolism $(15,16)$. Moreover, subcutaneous and visceral WAT express unique gene signatures (17). These data suggest that some of the distinct metabolic effects of subcutaneous and visceral WAT are cell autonomous. However, the molecular mechanisms governing the fat cell-intrinsic differences of these depots have not yet been established.

Brown fat-like adipocytes, having a multilocular morphology and expressing the brown adipocyte-specific uncoupling protein-1

Conflict of interest: The authors have declared that no conflict of interest exists. Citation for this article: J Clin Invest. 2011;121(1):96-105. doi:10.1172/JCI44271.
(Ucp1) protein, are found interspersed in the WAT of rodents and humans. These have been called adaptive or recruitable brown fat cells, brown in white (brite) cells, or beige cells (18-20). The development of these brown fat-like cells in WAT is dramatically enhanced during adaptation to cold or in response to treatment with $\beta 3$-selective adrenergic agonists (21-26). The brown-like transformation of WAT is most prominent in the inguinal subcutaneous depot, whereas the perigonadal adipose is less susceptible to browning $(21,23,27-30)$.

Recent studies have shown that Myf5-expressing embryonic progenitors give rise to the classic depots of brown adipose (31), but the brown-like cells induced by $\beta 3$-agonists in WAT do not appear to arise from this cell lineage. Various factors have been implicated in the activation of brown-like adipocyte development in WAT. Pharmacological activation of PPAR $\gamma$ by the antidiabetic thiazolidinedione drugs causes a "browning" of white adipose cells and tissue (19, 32-37). Moreover, transgenic expression of Foxc2 or increased prostaglandin levels sensitizes WAT to the effects of catecholamines and thereby promotes brown adipocyte development (38-40). Finally, a large number of knockout mouse strains that resist diet-induced obesity are characterized by a brown-like transformation of WAT (41-52). However, the transcriptional cascades that control the endogenous transformation of white to brown adipose tissues have not been determined.

Prdm16 is a transcriptional coregulator that controls the development of brown adipocytes in classic BAT depots (31, 53-56). Because the brown adipocytes in BAT and WAT belong to separate lineages, a role for Prdm16 in the adaptive induction of brown adipose within WAT has not been addressed. Furthermore, a detailed examination of Prdm16 expression in different depots of WAT is lacking. We previously reported that Prdm16 
A

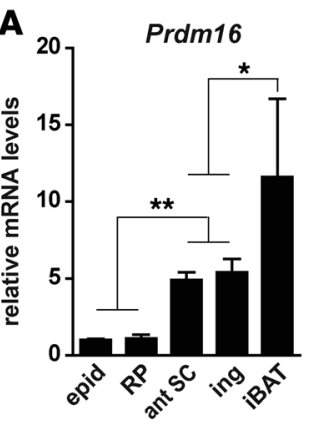

D

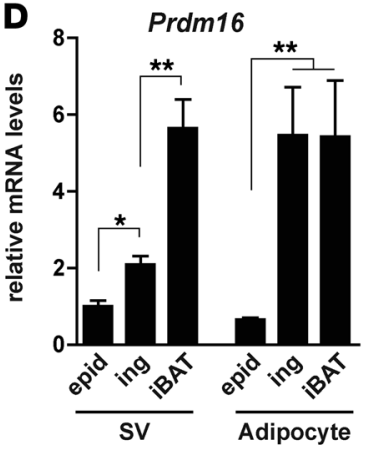

B

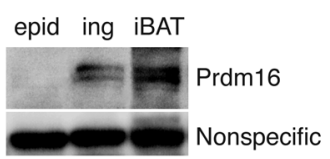

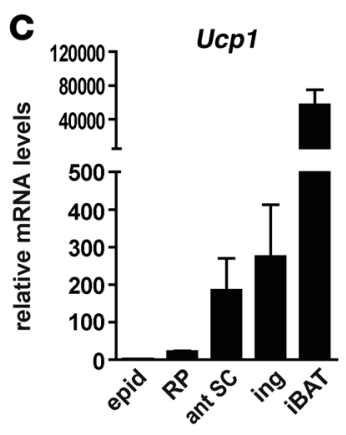

Glut4
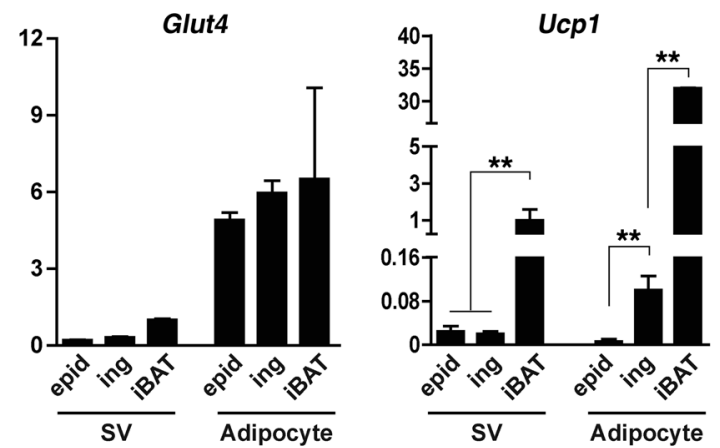
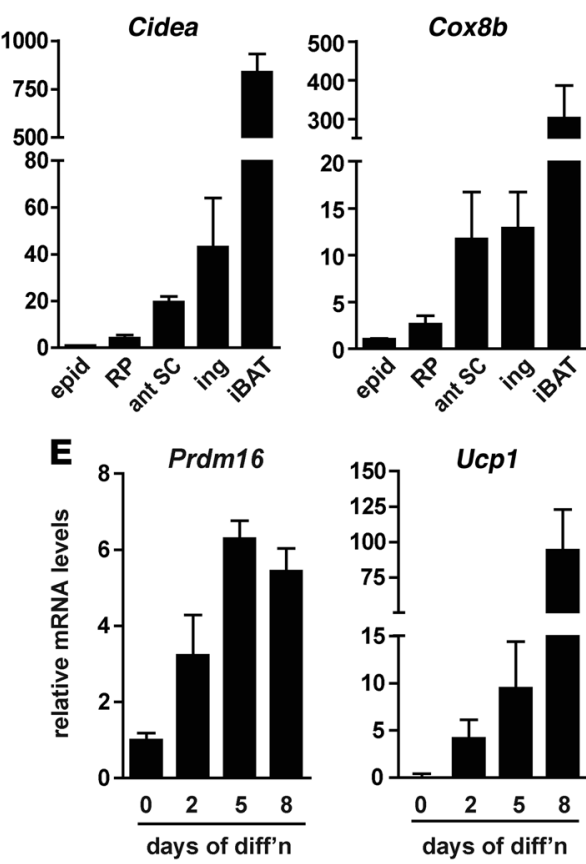

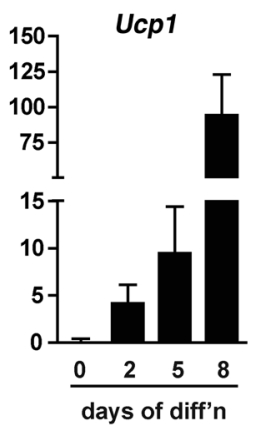

Figure 1

Expression of Prdm16 and a brown fat-like gene program in subcutaneous adipocytes. (A and B) Analysis of Prdm16 mRNA (A) and protein levels (B) in the indicated adipose depots from 12-week-old male WT mice. epid, epidWAT; RP, retroperitoneal WAT; ant SC, anterior (forelimb level) subcutaneous WAT; ing, ingWAT (subcutaneous). (C) mRNA levels of brown fat-selective (and Prdm16 target) genes (Ucp1, Cidea, and Cox8b) in adipose depots from A. (D) mRNA levels of Prdm16, Glut4 (mature adipocyte marker), and Ucp1 (brown adipocyte selective) in the SV and adipocyte fractions of epidWAT, ingWAT, and iBAT. (E) Prdm16 and Ucp1 mRNA levels during the in vitro differentiation of primary preadipocytes (from the SV fraction) of ingWAT. Values are mean $\pm \operatorname{SD}\left(n=4-6\right.$ mice per group). ${ }^{\star} P<0.05,{ }^{* \star} P<0.01$.

was expressed in BAT but not in WAT; however, we had only examined epididymal WAT (epidWAT) depots. In the present study, we showed that Prdm16 is highly expressed in subcutaneous WAT relative to its levels in epidWAT and retroperitoneal WAT. Transgenic expression of Prdm16 in all adipose tissues caused a selective transformation of subcutaneous WAT to a brown-like phenotype. The development of brown-like cells in the subcutaneous adipose of $a P 2-\operatorname{Prdm} 16$ animals was associated with a rise in whole-body energy expenditure and a suppression of weight gain in response to a high-fat diet. Importantly, $\operatorname{Prdm} 16$ was required for the induction of a thermogenic gene program in isolated, WT subcutaneous adipocytes and in vivo. Thus, our results identify Prdm16 as a critical mediator of adaptive thermogenesis in subcutaneous WAT.

\section{Results}

Prdm16 is highly expressed in subcutaneous white adipocytes. We originally identified Prdm16 as a transcriptional regulator that is selectively expressed in interscapular BAT (iBAT) versus epidWAT of mice (53). Here we performed a more detailed analysis of $\operatorname{Prdm} 16$ expression levels in different depots of WAT from mice housed at a standard temperature $\left(24^{\circ} \mathrm{C}\right)$. Importantly, $\operatorname{Prdm} 16$ mRNA was enriched by approximately 5 -fold in 2 different subcutaneous WAT depots, inguinal WAT (ingWAT) and anterior subcutaneous WAT, relative to its levels in epidWAT and retroperitoneal WAT, 2 prominent intra-abdominal WAT depots in male mice (Figure 1A). The Prdm16 mRNA level in subcutaneous WAT was approximately half that in iBAT. Moreover, Western blot analysis showed that Prdm 16 protein was present in ingWAT at about half its level in iBAT, and was not detectable in epidWAT (Figure 1B). Other brown adipocyte-selective (and Prdm16 target) genes, including Ucp1, Cidea, Cox8b, and Elovl3 (Figure 1C and Supplemental Figure 1A; supplemental material available online with this article; doi:10.1172/JCI44271DS1), were also substantially enriched in subcutaneous WAT compared with their levels in epidWAT and retroperitoneal WAT. Conversely, Retn, a gene known to be preferentially expressed in white versus brown adipose cells in mice $(37,53,57)$, was expressed at reduced levels in subcutaneous WAT compared with epidWAT and retroperitoneal WAT (Supplemental Figure 1A).

WAT is composed of mature adipocytes as well as other cells in what is termed the stromal-vascular (SV) fraction; the SV fraction contains preadipose cells, fibroblasts, immune cells, and blood vessel-associated cells. To determine which cell populations in subcutaneous WAT expresses Prdm16, we measured Prdm16 mRNA levels in fractionated adipose tissues. Prdm16 mRNA was significantly enriched in the mature adipocyte component of ingWAT relative to its levels in the SV fraction. In fact, Prdm16 mRNA levels in subcutaneous adipocytes were similar to that found in mature brown fat cells from iBAT. As expected, Glut4 and Ucp1, genes known to be selectively expressed in mature adipocytes, were coenriched with Prdm16 in the adipocyte fraction relative to the SV fraction (Figure 1D). The relatively high levels of Ucp1 mRNA in the SV component of iBAT was due to the presence of a substantial number of brown adipocytes that do not contain enough lipids to float during the cell fractionation process. Prdm16, Ucp1, and other brown-selective markers were also induced during the in vitro differentiation of pre- 

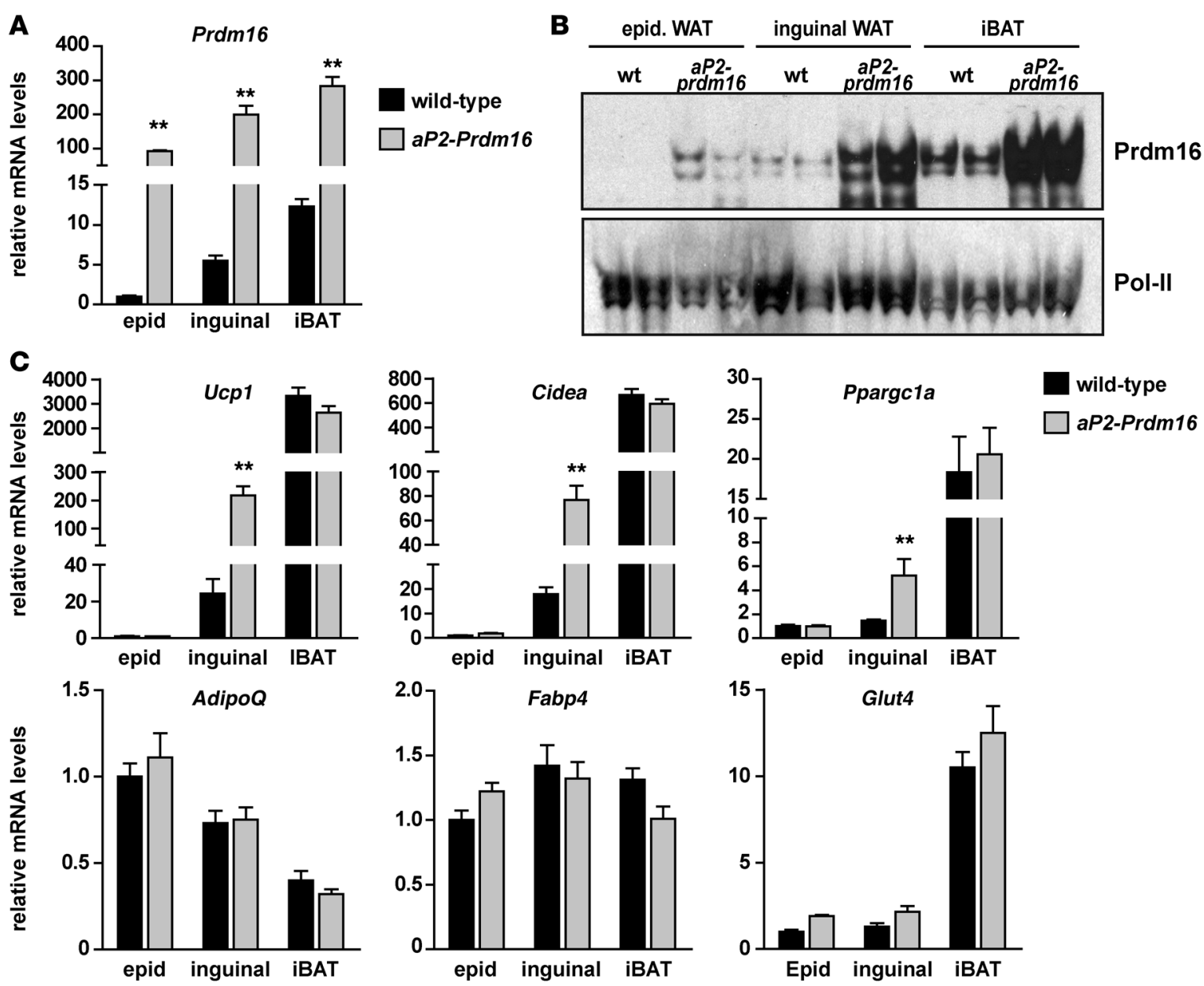

Figure 2

Transgenic expression of Prdm16 induces a thermogenic gene program in subcutaneous WAT. (A-C) The aP2 promoter/enhancer was used to drive ectopic Prdm16 expression in all adipose depots. (A) Real-time PCR analysis of Prdm16 mRNA levels in the epidWAT, ingWAT, and iBAT of 10- to 14-week-old male WT and aP2-Prdm16 mice fed a regular chow diet. (B) Western blot analysis of Prdm16 protein levels in adipose depots from mice in A. Pol-Il was used as a loading control. (C) mRNA levels of brown fat-selective genes (Ucp1, Cidea, and Ppargc1a) and general adipocyte markers (AdipoQ, Fabp4, and Glut4) in the indicated adipose depots from WT and aP2-Prdm16 mice. Values are mean \pm SEM ( $n=8$ mice per group). ${ }^{\star \star} P<0.01$ vs. WT.

adipocytes isolated from the SV fraction of ingWAT (Figure 1E and Supplemental Figure 1B), which indicates that a brown fat-like gene program is a cell-autonomous feature of subcutaneous adipocytes. Taken together, these results demonstrate that Prdm16 is expressed at very significant levels in mature subcutaneous adipocytes.

Transgenic expression of Prdm16 stimulates brown adipocyte development preferentially in subcutaneous WAT. We previously showed that transgenic expression of Prdm16 in all adipose depots (using the $-5 \mathrm{~kb}$ aP2 promoter/enhancer; also referred to as Fabp4) caused no morphological or molecular changes in the iBAT or WAT of mice under basal conditions (53). However, we only examined visceral epidWAT in these studies. The depot-selective expression of Prdm16 in subcutaneous WAT (Figure 1) strongly suggested reexamining these mice, especially with regard to the subcutaneous WAT depots. As shown in Figure 2A, 10- to 14-week-old male aP2-Prdm16 transgenic mice fed a regular chow diet and housed at $24^{\circ} \mathrm{C}$ expressed similar amounts of Prdm16 mRNA in epidWAT, ingWAT, and iBAT. Strikingly, Prdm 16 protein was elevated to a much greater extent in the ingWAT and iBAT compared with the epidWAT of these mice
(Figure 2B). Ectopic expression of Prdm16 robustly increased the levels of several brown fat-selective genes, such as Ucp1, Cidea, and Ppargc1a, by approximately 5- to 10-fold in ingWAT, but not epidWAT (Figure 2C). In female transgenic mice, Prdm16 increased the levels of brown fat-selective genes in ingWAT, but not in periovarian (gonadal) WAT (Supplemental Figure 2). Importantly, the expression levels of these genes in the male or female iBAT, which was already quite high in WT animals, were not increased in aP2-Prdm16 mice. Furthermore, ectopic expression of Prdm16 did not increase the levels of adipocyte selective genes common to white and brown adipocytes, such as AdipoQ, Fabp4, or Glut4, in any male or female depot examined (Figure 2C and Supplemental Figure 2). Therefore, Prdm16 preferentially induces a brown fat-like gene program in subcutaneous WAT.

The activation of brown adipose-selective genes in the subcutaneous WAT of aP2-Prdm16 animals was accompanied by a profound morphological transformation toward a BAT-like phenotype. Specifically, the ingWAT of aP2-Prdm16, but not WT littermates, contained numerous clusters of Ucp1-expressing, 

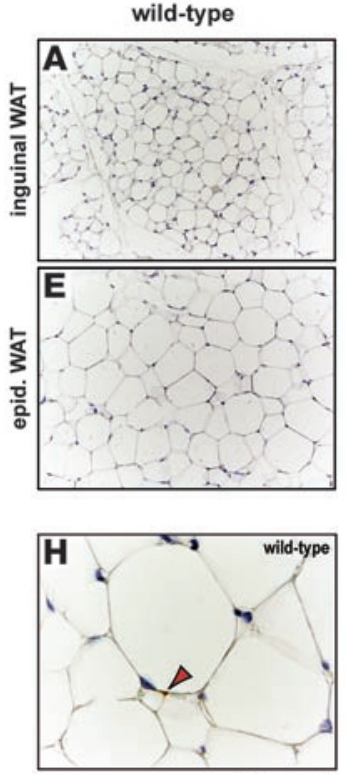

anti-Tyrosine Hydroxylase
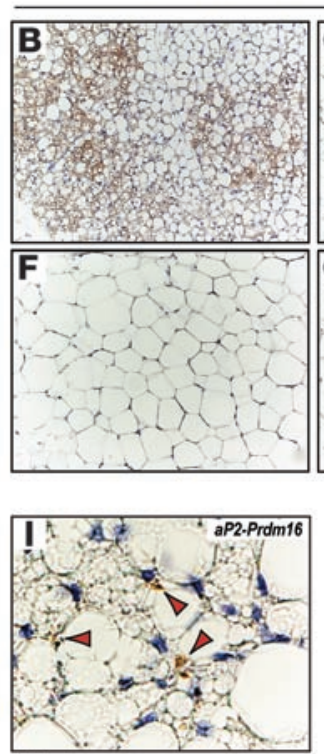

aP2-Prdm16
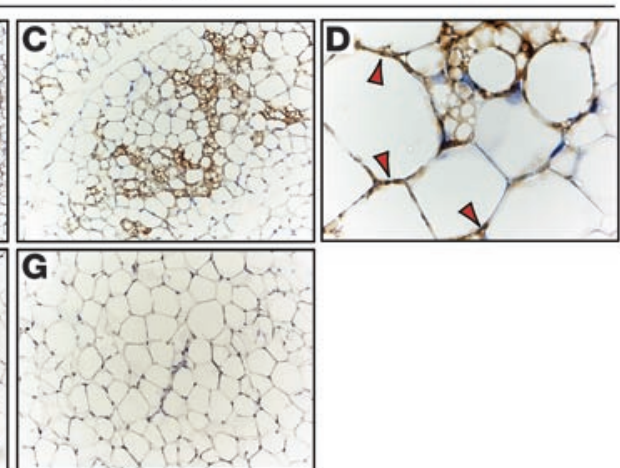

$\mathrm{TH}+$ fibers $/ 100$ adipocytes

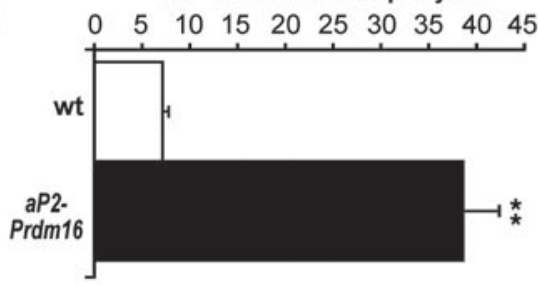

Figure 3

Prdm16 stimulates the development of brown-like adipocytes in subcutaneous WAT. (A-G) Immunohistochemistry for Ucp1 protein (brown stain) in sections of ingWAT (A-D) and epidWAT (E-G) from 10- to 14-week-old male WT (A and E) and aP2-Prdm16 (B-D, F, and G) mice. (D) High-magnification and representative image of ingWAT from a transgenic animal. Arrowheads depict Ucp1 immunopositive cells that have a unilocular morphology typical of white adipocytes. ( $H$ and $\mathbf{I})$ Immunohistochemistry for TH protein in samples of ingWAT from WT (H) and aP2-Prdm16 (I) animals as described above. Original magnification, $\times 20(\mathbf{A}-\mathbf{C}$ and $\mathbf{E}-\mathbf{G}) ; \times 100(\mathbf{D}, \mathbf{H}$, and I). (J) Quantification of TH-expressing nerve fibers in WT and transgenic ingWAT. Values are mean \pm SEM $(n=20$ fields per sample in each of 3 animals per group). ${ }^{\star \star} P<0.01$ vs. WT.

multilocular adipocytes under basal conditions (Figure 3, A-D). However, Ucp1-expressing adipocytes were not detected in the epidWAT from transgenic or WT mice (Figure 3, E-G). Furthermore, we did not observe any discernible morphological changes in the iBAT between transgenic and WT mice (Supplemental Figure 3). Interestingly, aP2-Prdm16 mice ingWAT contained 5 times more tyrosine hydroxylase-positive $\left(\mathrm{TH}^{+}\right)$, sympathetic parenchymal nerve fibers than that of WT littermates. The increase in sympathetic nerve fibers was depot selective, since we did not observe any change in the number of $\mathrm{TH}^{+}$parenchymal fibers between the epidWAT of transgenic and WT animals. Together, these results indicate that Prdm 16 can drive a full program of brown adipocytelike development, obvious at both morphological and molecular levels, in subcutaneous WAT under basal conditions.

Protection against high-fat diet-induced weight gain in aP2-Prdm 16 mice. In light of the striking effect of Prdm16 action in subcutaneous WAT depots, the transgenic animals were subjected to metabolic analyses in response to both regular chow and high-fat diet. On regular chow, aP2-Prdm16 mice and their WT littermates did not display any notable difference in daily energy balance, but aP2-Prdm16 mice had a slightly increased lean mass (Supplemental Figure 4). During high-fat feeding, aP2-Prdm16 mice gained significantly less weight than their age- and sex-matched WT littermates (Figure 4A). Body composition analyses after 16 weeks of high-fat diet showed that transgenic animals had gained substantially less fat, resulting in a higher lean/fat mass ratio compared with WT animals (Figure 4B). The suppressed weight gain in the by insulin tolerance tests (Figure 4E). As was observed in chowfed animals, Ucp1-expressing fat cells (both multilocular and unilocular) were readily detected in the ingWAT of aP2-Prdm 16 mice, but not in WT mice, after 16 weeks of consuming a high-fat diet (Figure 4F). The WAT of high-fat-fed aP2-Prdm 16 animals also expressed higher levels of brown fat-selective genes relative to WT mice (Figure 4F). Interestingly, high-fat diet promoted a significant induction in the levels of thermogenic genes in epidWAT of aP2-Prdm16 animals (Figure 4F); this effect was not seen in chow-fed cohorts. Importantly, the levels of these thermogenic genes were not altered by the aP2-Prdm 16 transgene in the iBAT, even after 16 weeks of high-fat diet. These results suggest that the elevated energy dissipation in aP2-Prdm16 animals is not due to superactivation of the endogenous classical BAT. Rather, it is more likely to have occurred via the thermogenic program expressed in the remodeled brown-like subcutaneous adipose. Together, these observations demonstrate that increased Prdm 16 action in adipose tissue raises energy expenditure to counteract weight gain and drive glucose disposal. These actions appear to occur mainly in the subcutaneous WAT.

Prdm16 is a cell-antonomous regulator of thermogenesis in subcutaneous adipocytes. The transgenic experiments above showed that elevated Prdm16 expression in WAT can drive a program of adaptive thermogenesis in vivo. However, a key question is whether isolated subcutaneous adipocytes require Prdm 16 for thermogenic gene expression and respiratory function. To answer this question, we knocked down expression of Prdm16 in primary cultured adipocytes from 

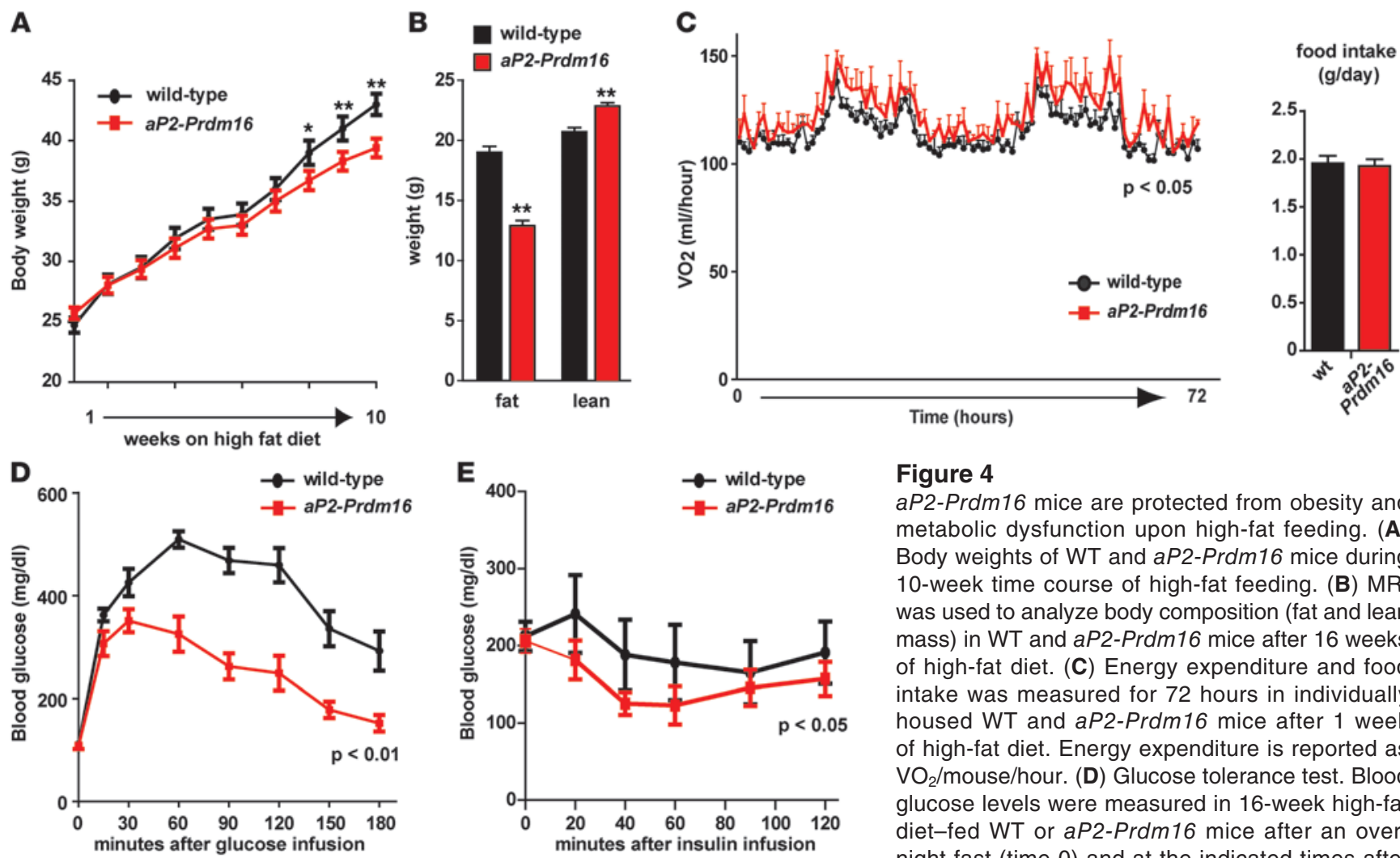

Figure 4

aP2-Prdm16 mice are protected from obesity and metabolic dysfunction upon high-fat feeding. (A) Body weights of WT and aP2-Prdm16 mice during 10-week time course of high-fat feeding. (B) MRI was used to analyze body composition (fat and lean mass) in WT and aP2-Prdm16 mice after 16 weeks of high-fat diet. (C) Energy expenditure and food intake was measured for 72 hours in individually housed WT and aP2-Prdm16 mice after 1 week of high-fat diet. Energy expenditure is reported as $\mathrm{VO}_{2} /$ mouse/hour. (D) Glucose tolerance test. Blood glucose levels were measured in 16-week high-fat diet-fed WT or aP2-Prdm16 mice after an overnight fast (time 0 ) and at the indicated times after intraperitoneal injection of glucose. (E) Insulin tolerance test. Blood glucose levels were measured after an overnight fast (time 0 ) and at the indicated times after an intraperitoneal injection of insulin in mice from D. (F) Immunohistochemistry for Ucp1 (brown stain) protein in ingWAT from WT and aP2-Prdm16 mice after 16 weeks of high-fat diet. The boxed region is shown at higher magnification at right. Ucp1-expressing multilocular and unilocular fat cells are indicated by the arrow and arrowhead, respectively. Original magnification, $\times 20$ (left and middle); $\times 100$ (right). mRNA levels of brown fat-selective genes (Ucp1 and Cidea) were determined in epidWAT, ingWAT, and iBAT from WT and aP2-Prdm16 animals after 16 weeks of high-fat diet. Significance between curves was determined by 2-way ANOVA. Values are mean \pm SEM $(n=16$ mice per group per experiment). ${ }^{\star} P<0.05,{ }^{\star \star} P<0.01$ vs. WT.

subcutaneous WAT of WT mice using a shRNA expressed from an adenovirus. Adenoviral vectors expressing a control scrambled sequence or Prdm16 shRNA (sh-Prdm16) were used to infect subconfluent SV cells from ingWAT, which contain a high proportion of adipocyte precursors. These SV cultures were induced to undergo adipogenesis 2 days after adenovirus transduction. As shown in Figure 5A, endogenous Prdm16 mRNA levels were very efficiently decreased by the sh-Prdm16-expressing vector in adipocytes. Moreover, the adenoviral vectors (which also expressed GFP) were taken up and expressed by greater than $90 \%$ of adipocytes derived from primary inguinal preadipose cells (Figure 5B). Knockdown of Prdm16 did not interfere with adipogenesis per se, since control and Prdm16-depleted cells underwent a similar extent of morphological differentiation, as shown by Oil-Red-O staining for lipid accumulation (Figure 5C). Control and Prdm16-depleted adipocyte cultures also expressed equivalent levels of general adipocyte-related mRNAs like Fabp4 and AdipoQ (Figure 5C). However, suppression of Prdm16 expression completely blocked the differentiation-linked expression of most brown fat-selective genes, including Ucp1, Cidea, and Cox8b (Figure 5D). This decrease in Prdm16 levels also blunted the activation of thermogenic genes Ucp1 and Ppargc1a in response to the synthetic catecholamine isoproterenol. Western blot analysis indicated that Ucp1 protein expression was almost completely ablated by loss of Prdm16 in both untreated and isoproterenol-stimulated adipocytes (Figure 5E). To determine whether Prdm16 is required for the expression of brown fat-selective genes in mature fat cells or whether it functions during the process of differentiation, we knocked down Prdm16 in adipocytes 


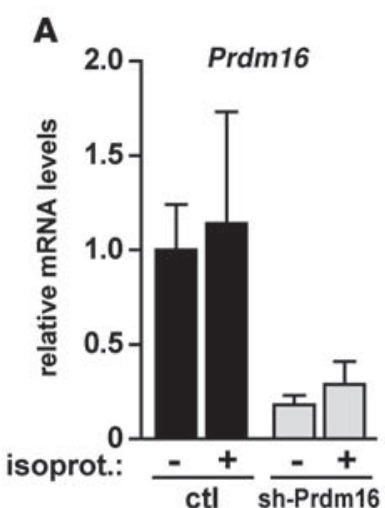

D

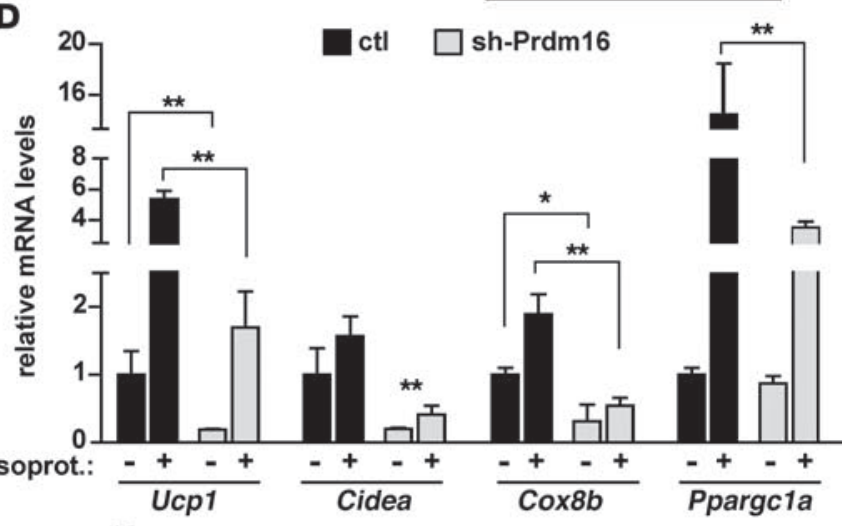

$\mathbf{F}$

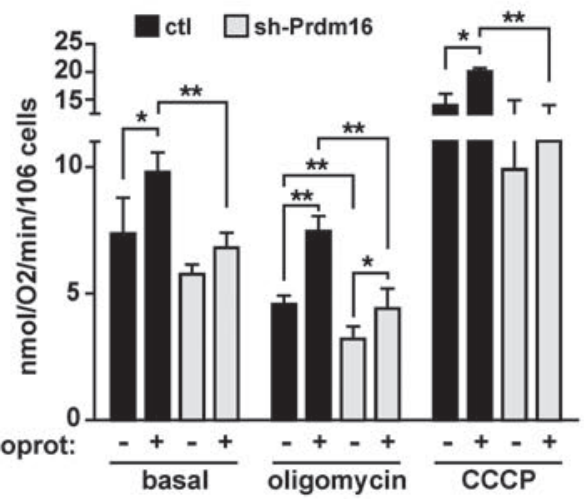

G

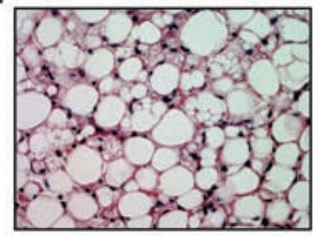

wild-type
B
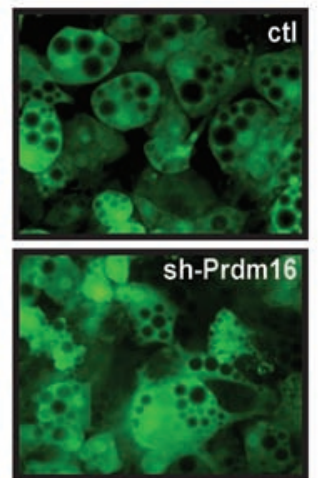

sh-Prdm16

H

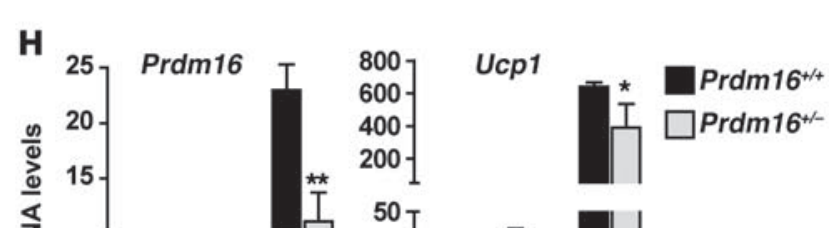

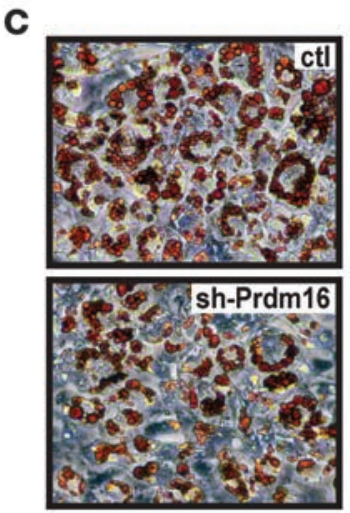

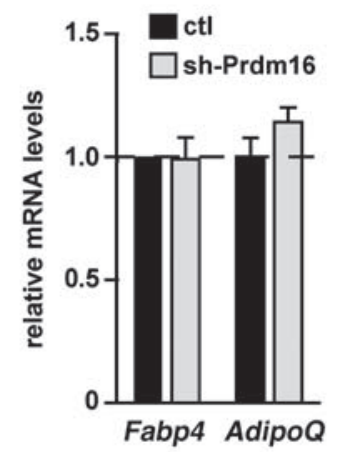

E
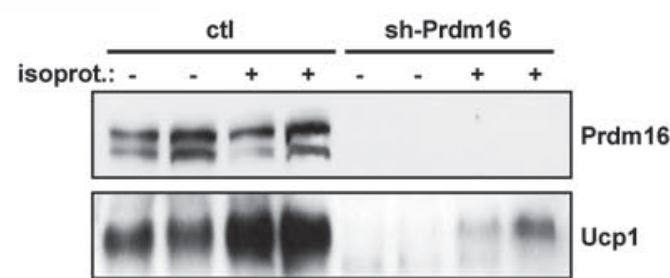

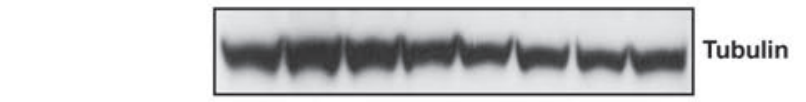
rdm16
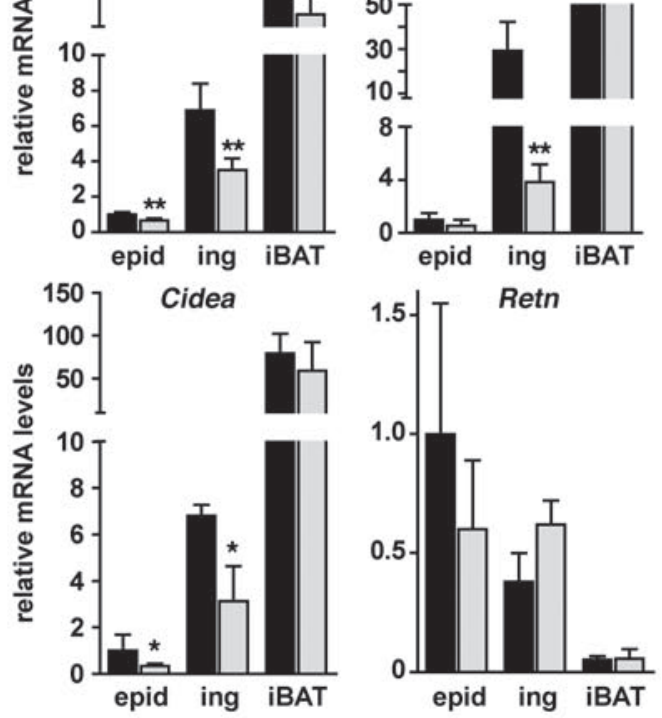

Figure 5

Prdm16 is required for expression of a thermogenic gene program in subcutaneous adipocytes. (A-G) Subcutaneous preadipocytes in the SV fraction of inguinal fat from WT mice were transduced with adenovirus expressing a shRNA targeted to Prdm16 or a scrambled control shRNA (ctl). These cultures were then induced to differentiate in vitro into adipocytes. (A) Prdm16 mRNA levels (with and without isoproterenol stimulation, as indicated). (B) GFP was expressed from adenoviral shRNA vectors, and its expression was used to reveal control shRNA-and sh-Prdm16-transduced adipocytes. (C) Oil-Red-O staining (red) for lipid accumulation. mRNA levels of general adipocyte markers (Fabp4 and AdipoQ) were also determined. (D) mRNA levels of brown fat-selective genes (Ucp1, Cidea, Cox8b, and Ppargc1a). (E) Western blot analysis for Prdm16 and Ucp1 protein. (F) Oxygen consumption, assayed using a Clark-type electrode. Oligomycin (ATPase inhibitor) and CCCP (chemical uncoupler) were added to cells to measure the rates of uncoupled and maximal respiration, respectively. (G and H) WT Prdm16 ${ }^{+/+}$and heterozygous Prdm $16^{+/-}$littermates were treated with CL316,243 for 3 days. (G) H\&E staining of inguinal adipose tissue. (H) mRNA levels of Prdm16, brown fat-selective genes Ucp1 and Cidea, and Retn in epidWAT, ingWAT, and iBAT. Original magnification, $\times 20(\mathbf{B}, \mathbf{C}$, and $\mathbf{G})$. Values are mean $\pm \mathrm{SD}(n=3-5)$. ${ }^{*} P<0.05$; ${ }^{* *} P<0.01$. 
7 days after induction of differentiation. Loss of Prdm16 from fully differentiated adipocytes caused a dramatic reduction in the expression of brown fat-related genes like Ucp1, Cidea, Ppargc1a, and $C o x 8 b$ without affecting the levels of general fat markers Fabp4 and AdipoQ (Supplemental Figure 6). These results indicate that Prdm16 is required in mature subcutaneous fat cells for the full activation of a brown fat-like gene program.

Next, to examine whether Prdm16 is required for the respiratory activity of the isolated primary subcutaneous adipocytes, we measured oxygen consumption in control and Prdm16-depleted cells using a Clark electrode. sh-Prdm16-expressing subcutaneous adipocyte cultures had a significantly lower rate of uncoupled (state IV) respiration under unstimulated conditions. Upon treatment with isoproterenol, control cultures underwent a dramatic increase in uncoupled respiration, whereas adipocytes with reduced Prdm16 had a severely blunted response. Moreover, Prdm16-depleted cells exhibited lower maximal respiration than control cells after treatment with the uncoupler CCCP (Figure 5F). These results demonstrate that $\operatorname{Prdm} 16$ stimulates uncoupled respiration in a $\beta$-agonist-dependent manner in subcutaneous adipocytes.

Finally, we tested the in vivo requirement for Prdm 16 expression in the $\beta$-adrenergic-induced activation of a brown fat gene program in WAT. Prdm16-deficient mice have a cleft palate and die perinatally, thus precluding analysis of Prdm16-deficient WAT (60). We have therefore studied the phenotype of WAT from 3-monthold male WT and $\operatorname{Prdm} 16^{+/-}$heterozygous littermates after 3 days of treatment with the $\beta 3$-selective adrenergic agonist CL316,243. As shown in Figure 5G, there were many fewer clusters of multilocular brown fat-like areas in the ingWAT of $\mathrm{Prdm} 16^{+/-}$compared with WT mice. At the molecular level, approximately $50 \%$ reduction in Prdm 16 mRNA levels causes a significant and specific reduction in the expression of brown fat-selective genes like Ucp1 and Cidea in WAT, especially in the ingWAT depot (Figure 5H). There was a near 8-fold decrease in Ucp1 levels in subcutaneous ingWAT from $\mathrm{Prdm} 16^{+/-}$compared with WT animals. Conversely, Retn, a white fat-enriched gene, tended to be increased in the ingWAT of Prdm $16^{+/-}$relative to WT mice. Interestingly, the expression of BAT-selective genes in the inguinal depot was much more sensitive to Prdm16 dosage compared with iBAT, at least under $\beta 3$-agonist-stimulated conditions. These results reveal a genetic requirement for Prdm 16 in the $\beta 3$-adrenergic-stimulated induction of a brown fat phenotype within WAT.

\section{Discussion}

Prdm16 is a key transcriptional regulator of the cellular lineage that gives rise to the classic brown adipose depots in mice. We report here that Prdm16 was expressed at substantial levels in subcutaneous WAT, where it regulated a functional thermogenic gene program. Thus, the Prdm16 pathway is a conserved mechanism that controls brown adipocyte development and thermogenic gene expression in 2 separate cell lineages.

The cellular precursor cell types for brown fat-like adipocytes in WAT are unknown. Notably, Prdm16 was highly expressed by mature adipocytes in subcutaneous WAT. Moreover, Prdm16 drove brown fat-like cell development in WAT when ectopically expressed in adipocytes from a differentiation-selective promoter. Finally, Prdm16 was cell-autonomously required in mature fat cells for induction of a brown fat-selective gene program. Together, these results suggest that mature adipocytes can be stimulated to express a brown fat-like phenotype, at least in ingWAT. Spe- cific markers for white adipocytes are needed to answer whether brown fat-like cells can arise by transdifferentiation. This does not exclude the existence of alternative or additional progenitor cell types for the brown fat-like cells in WAT, including fibroblasts or preadipocytes, as has been proposed previously by us and many others. Additional marker genes and fate-mapping experiments are needed to resolve this issue.

Transgenic expression of Prdm16 in fat tissues stimulated the development of Ucp1-expressing, brown-like fat cells in ingWAT, but not epidWAT. Notably, there was a significant increase in the levels of thermogenic genes in the absence of morphological brown-like adipose development in the epidWAT of transgenic mice, although the absolute level of thermogenic gene expression was far lower in the epididymal than in the inguinal depot. Moreover, haploinsufficiency of Prdm 16 caused a pronounced reduction in the expression of brown fat-specific genes in WAT, particularly in the subcutaneous depot, after $\beta 3$-adrenergic stimulation. Thus, the extent of thermogenic gene induction in WAT is correlated with Prdm16 levels. The higher density of sympathetic nerve endings in the inguinal relative to the epididymal depot (61) presumably amplifies the browning effects of ectopic Prdm16 expression. Consistent with this, brown adipocytes can be induced in the epididymal depots of transgenic animals in response to a strong pharmacological $\beta 3$-adrenergic stimulus (53). Although ectopic Prdm16 mRNA was equally increased in ingWAT and epidWAT of transgenic mice, the protein preferentially accumulated in the ingWAT depot. It is likely that epidWAT expresses a factor that degrades Prdm16, or affects its translation, to limit conversion of this tissue into brown-like fat. Based on its relatively large mass and increased expression of a brown fat-like gene program, it is likely that subcutaneous depots account for a substantial portion of the thermogenic output from WAT. However, we do not exclude a role for visceral depots in the thermogenic response to cold or high-fat diet, especially the depots that have propensity to develop brown-like adipocytes, like the mesenteric WAT (30). The relative contribution of WAT to total Ucp1-mediated energy dissipation, much of which likely occurs in classic BAT depots, remains to be determined.

Prdm 16 induces the formation of brown fat-like cells in WAT under basal conditions, but the thermogenic function of the transgenic WAT was only revealed when another stimulus was given in this case, a high-fat diet. The ability of overfeeding to elicit sympathetic outflow to brown fat and heart is well known (62-65). Therefore, we speculate that $\operatorname{Prdm} 16$ acts as a determining factor in subcutaneous adipocytes to establish a gene program under basal conditions that can allow for activation of thermogenesis in response to cold or overfeeding. Although these stimuli did not increase Prdm 16 levels, further studies are needed to assess whether catecholamines can directly or indirectly enhance Prdm16 activity. Importantly, a program of adaptive thermogenesis in WAT of Prdm16 transgenic animals suppressed obesity caused by a highfat diet. These mice also displayed a dramatic enhancement in the clearance of blood glucose relative to WT mice after intraperitoneal glucose infusion. This effect reflects enhanced insulin action, much of which is likely secondary to the reduced obesity and fat mass in transgenic mice, but it may also involve non-insulin-dependent glucose disposal into the transgenic WAT. Additional metabolic analyses of these mice are needed to answer this question.

In classic brown fat depots, thermogenesis is tightly regulated by catecholamines secreted by sympathetic nerve endings in BAT. Moreover, the density of brown-like adipocytes in WAT depots is 
positively correlated with the number of sympathetic nerve fibers (61). The clusters of brown fat-like cells that appear in $\operatorname{Prdm} 16$ transgenic WAT may develop at and/or near sympathetic nerve terminals. If so, it may be that all transgenic adipocytes are competent to induce a brown fat program, but require an additional sympathetic stimulus. Alternatively, distinct subtypes of subcutaneous adipocytes exist, with different propensities to acquire brown fat-like features in response to Prdm16 expression. Notably, we observed a dramatic increase in the number of sympathetic parenchymal nerve fibers infiltrating the ingWAT of aP2-Prdm16 transgenic mice compared with WT animals (Figure 3). How $\operatorname{Prdm} 16$ acts to stimulate the recruitment of sympathetic nerves to WAT is unknown, but of considerable interest. The increase in Prdm16 expression in epidWAT of transgenic mice may have been insufficient to stimulate nerve recruitment. Alternatively, other factors unique to subcutaneous depots may act in parallel with Prdm 16 to fully stimulate brown fat cell development. Whatever the mechanism, the enrichment of sympathetic nerves in the vicinity of brown fat-like cells in WAT ensures that the tissue can efficiently undergo thermogenesis in response to the activity of the sympathetic nervous system. The sympathetic nervous system undoubtedly provides a major control mechanism for the thermogenesis of subcutaneous WAT in vivo. However, cultured subcutaneous adipocytes from WT mice expressed a differentiationlinked brown fat-like gene program ex vivo; this gene profile was intrinsic to subcutaneous adipocytes and thus not obligatorily linked to the sympathetic nervous system. Importantly, Prdm16 was cell-autonomously required for the expression of this thermogenic gene program in subcutaneous adipocytes.

It will now be important to carefully examine whether subcutaneous human WAT not chronically stimulated by cold exposure also expresses Prdm16 at reasonable levels. Interestingly, biopsies of adult human BAT contain an admixture of unilocular and multilocular adipocytes that express enriched amounts of Prdm16 mRNA (66-69); the morphological appearance of this tissue is reminiscent of subcutaneous adipose (beige fat) from cold-exposed or $\beta 3$ agonist-treated rodents. Therefore, a key question is whether human BAT is analogous to the classic BAT or adaptive BAT in rodents.

It is still debated whether the amount of activated BAT in humans is sufficient to impact energy balance in a meaningful way. On the other hand, subcutaneous WAT is very abundant in humans. A recent report suggests that thermogenic, Ucp1-expressing beige fat cells in human subcutaneous fat correlates with insulin sensitivity (70). If subcutaneous human WAT expresses Prdm16, this tissue could be a promising target for stimulating energy expenditure pathways to counteract obesity and insulin resistance.

\section{Methods}

Mice and dissections. All animal experiments were performed according to procedures approved by the Institutional Animal Care and Use Committee of Dana-Farber Cancer Institute or of University of Pennsylvania. Mice were either maintained on a standard rodent chow or a $60 \%$ high-fat diet (Research Diets) with 12-hour light and dark cycles. The derivation and initial characterization of aP2-Prdm16 transgenic mice was described previously (53). However, for this study, we backcrossed the aP2-Prdm16 strain into the $\mathrm{C} 57 \mathrm{BL} / 6$ genetic background for 8 generations to enable high-fat diet-induced obesity studies. Prdm16-deficient mice, which have been described previously (60), were provided by B.C. Bjork and D.R. Beier (Harvard Medical School, Boston, Massachusetts, USA). Unless noted specifically, male animals were used for experiments. In general, we adhered to the nomenclature for dissected depots described by Murano et al. (30). Specific definitions of dissected depots are as follows: epidWAT (or perigonadal depot in females), prominent bilateral intra-abdominal visceral depots in male mice attached to the epididymides; periovarian WAT (or perigonadal depot in males), bilateral intra-abdominal visceral depots attached to the ovaries; retroperitoneal WAT, bilateral depots in abdominal cavity behind the peritoneum on the dorsal side of the kidney; anterior subcutaneous, bilateral superficial subcutaneous WAT between the skin and muscle fascia posterior to the lower segment of the upper limbs; ingWAT, bilateral superficial subcutaneous WAT depots between the skin and muscle fascia just anterior to the lower segment of the hind limbs; iBAT, most prominent depot of classic brown adipocytes in rodents, found as bilobed tissue between the scapulae. CL316,243 (Sigma-Aldrich) at $1 \mathrm{mg} / \mathrm{kg}$ was injected intraperitoneally into mice. All metabolic and gene expression studies were performed in adult (8- to 16-week-old) male mice of the C57BL/6 genetic background (Taconic).

Real-time PCR analysis and Western blotting. Total RNA from cultured cells was isolated using the TRIzOL method (Invitrogen) combined with Qiagen RNEasy mini columns according to manufacturer's instruction. For real-time PCR analysis, RNA was reverse transcribed using the ABI high capacity CDNA synthesis kit and used in quantitative PCR reactions containing SYBR-green fluorescent dye (ABI). Relative expression of mRNAs was determined after normalization with TBP levels using the ${ }^{\Delta \Delta} \mathrm{Ct}$ method. Quantitative PCR was performed using the ABI-7600HT PCR machine. Primer sequences were reported previously (53). Student's $t$ test was used to compare expression levels and obtain statistics. Primers used for real-time PCR are shown in Supplemental Table 1. For Western blot analysis, cells or tissues were lysed in RIPA buffer ( $0.5 \%$ NP- $40,0.1 \%$ sodium deoxycholate, $150 \mathrm{mM} \mathrm{NaCl}, 50 \mathrm{mM}$ Tris-Cl, $\mathrm{pH}$ 7.5). Lysates were resolved by SDS-PAGE, transferred to PVDF membrane (Millipore), and probed with anti-Ucp1 (Chemicon), anti-Prdm16 (rabbit polyclonal), and anti-pol-II (Santa Cruz Biotechnology).

Cell culture. Primary white fat stromal vascular and mature fat cells were fractionated according to published methods $(71,72)$. Primary SV cells were cultured in DMEM/F12 containing 10\% FBS. For adenoviral infection of primary SV cells, $70 \%$ confluent cell cultures were incubated with sh-Prdm16- or scrambled shRNA-expressing adenovirus (MOI 50) overnight in growth medium. The medium was then replaced, and cells were maintained in growth medium for an additional 24 hours before inducing adipogenic differentiation. Adipocyte differentiation was induced in preadipocytes cultures by treating confluent cells for 48 hours in medium containing $10 \%$ FBS, $0.5 \mathrm{mM}$ isobutylmethylxanthine, $125 \mathrm{nM}$ indomethacin, $1 \mu \mathrm{M}$ dexamethasone, $850 \mathrm{nM}$ insulin, $1 \mathrm{nM} \mathrm{T}_{3}$, and $1 \mu \mathrm{M}$ rosiglitazone (Cayman Chemical). 2 days after induction, cells were switched to maintenance medium containing $10 \% \mathrm{FBS}, 850 \mathrm{nM}$ insulin, $1 \mathrm{nM} \mathrm{T}_{3}$, and $1 \mu \mathrm{M}$ rosiglitazone. To stimulate thermogenesis, cells were incubated with $10 \mu \mathrm{M}$ isoproterenol for 4 hours. All chemicals for cell culture were obtained from Sigma-Aldrich unless otherwise noted. GFP expressed from the adenoviral vectors was used to monitor infection efficiency, which was typically greater than $80 \%$.

Oxygen consumption assays. Primary SV cultures expressing control scrambled shRNA or sh-Prdm16 were induced to undergo adipogenesis. At day 8 of differentiation, oxygen consumption was measured in fat cells using a Strathkelvin Clark-type electrode. $1 \mu \mathrm{M}$ oligomycin (Sigma-Aldrich) or $2 \mu \mathrm{g}$ CCCP (Sigma-Aldrich) was added to block state III respiration and induce uncoupling, respectively.

Metabolic phenotyping. For glucose tolerance tests, animals were fasted overnight. The next morning, glucose levels in tail blood were measured with a standard glucometer prior to and at timed intervals following an 
intraperitoneal injection of $2 \mathrm{~g} / \mathrm{kg}$ D-glucose. Body weight was measured on a weekly basis for high-fat feeding experiments. Fat and fat-free mass was measured by MRI. Whole-body energy metabolism was evaluated using a Comprehensive Lab Animal Monitoring System (CLAMS, Columbia Instruments). Mice were acclimated in the metabolic chambers for 2 days before the starting the experiment to minimize stress from the housing change. $\mathrm{CO}_{2}$ and $\mathrm{O}_{2}$ levels were collected every 32 minutes for each mouse over a period of 3 days. Movement and food intake are measured more frequently at regular intervals.

Histological analysis. Tissues were dissected and fixed in $4 \%$ paraformaldehyde overnight and rinsed with phosphate-buffered saline before embedding in paraffin. For Ucp1 immunohistochemistry, paraffinembedded sections were incubated with anti-Ucp1 (Abcam), followed by detection using the $\mathrm{ABC}$ Vectastain-Elite kit (Vector Labs) according to the manufacturer's instructions. Immunohistochemistry for TH was performed using anti-TH antibodies (Millipore) as described previously (73). $\mathrm{TH}^{+}$parenchymal nerve fibers (i.e., fibers closely associated to adipocytes in the depots) were counted; perivascular fibers (those in contact with arterioles and venules) were not considered in the quantitative analysis. 20 randomly selected areas, representative of the depot, were studied in the inguinal subcutaneous depot of each animal using a Nikon Eclipse E800 light microscope with $\mathrm{a} \times 100$ oil immersion objective at $\times 1,250$ final magnification. The density of TH-immunoreactive fibers was calculated as the number of fibers per 100 adipocytes.
Statistics. Statistical significance was assessed by ANOVA, 2-tailed Student's $t$ test, or linear regression analysis using GraphPad Prism, as indicated. A $P$ value less than 0.05 was considered significant.

\section{Acknowledgments}

We are grateful to Bryan Bjork and David R. Beier for Prdm16-deficient mice. We thank Mary Selak and Nicolai Dolibai for assistance with oxygen consumption assays and Dina Laznik and Matthew Brown for technical assistance. This work was supported by NIH grant DK081605 and a Keystone Innovation Starter Kit (KISK) program grant to P. Seale; by NIH grant DK31405-24 to B.M. Spiegelman; and by the Institute for Diabetes, Obesity, and Metabolism (IDOM) at the University of Pennsylvania School of Medicine.

Received for publication July 8, 2010, and accepted in revised form October 13, 2010.

Address correspondence to: Patrick Seale, University of Pennsylvania, Clinical Research Building-727, 415 Curie Blvd., Philadelphia, Pennsylvania 19104, USA. Phone: 215.573.8856; Fax: 215.898.5408; E-mail: sealep@upenn.edu. Or to: Bruce M. Spiegelman, Dana-Farber Cancer Institute, 1 Jimmy Fund Way, Boston, Massachusetts 02115, USA. Phone: 617.632.3567; Fax: 617.632.5363; E-mail: bruce_spiegelman@dfci.harvard.edu.
1. Lloyd-Jones D, et al. Heart disease and stroke statistics--2009 update: a report from the American Heart Association Statistics Committee and Stroke Statistics Subcommittee. Circulation. 2009;119(3):e21-181.

2. Mann JI. Diet and risk of coronary heart disease and type 2 diabetes. Lancet. 2002;360(9335):783-789.

3. Wang Y, Rimm EB, Stampfer MJ, Willett WC, Hu FB. Comparison of abdominal adiposity and overall obesity in predicting risk of type 2 diabetes among men. Am J Clin Nutr. 2005;81(3):555-563.

4. Klein S, et al. Waist circumference and cardiometabolic risk: a consensus statement from shaping America's health: Association for Weight Management and Obesity Prevention; NAASO, the Obesity Society; the American Society for Nutrition; and the American Diabetes Association. Diabetes Care. 2007;30(6):1647-1652.

5. Fox CS, et al. Abdominal visceral and subcutaneous adipose tissue compartments: association with metabolic risk factors in the Framingham Heart Study. Circulation. 2007;116(1):39-48.

6. Despres JP, et al. The insulin resistance-dyslipidemic syndrome: contribution of visceral obesity and therapeutic implications. Int J Obes Relat Metab Disord. 1995;19 Suppl 1:S76-S86.

7. Ross R, Fortier L, Hudson R. Separate associations between visceral and subcutaneous adipose tissue distribution, insulin and glucose levels in obese women. Diabetes Care. 1996;19(12):1404-1411.

8. Rexrode KM, et al. Abdominal adiposity and coronary heart disease in women. JAMA. 1998; 280(21):1843-1848.

9. Porter SA, Massaro JM, Hoffmann U, Vasan RS, O’Donnel CJ, Fox CS. Abdominal subcutaneous adipose tissue: a protective fat depot? Diabetes Care. 2009;32(6):1068-1075.

10. Snijder MB, et al. Low subcutaneous thigh fat is a risk factor for unfavourable glucose and lipid levels, independently of high abdominal fat. The Health ABC Study. Diabetologia. 2005;48(2):301-308.

11. Miyazaki Y, et al. Effect of pioglitazone on abdominal fat distribution and insulin sensitivity in type 2 diabetic patients. J Clin Endocrinol Metab. 2002;87(6):2784-2791.

12. Kim JY, et al. Obesity-associated improvements in metabolic profile through expansion of adipose tissue. J Clin Invest. 2007;117(9):2621-2637.

13. Klein S, et al. Absence of an effect of liposuction on insulin action and risk factors for coronary heart disease. NEngl J Med. 2004;350(25):2549-2557.

14. Tanko LB, Bagger YZ, Alexandersen P, Larsen PJ, Christiansen C. Peripheral adiposity exhibits an independent dominant antiatherogenic effect in elderly women. Circulation. 2003;107(12):1626-1631.

15. Hocking SL, Chisholm DJ, James DE. Studies of regional adipose transplantation reveal a unique and beneficial interaction between subcutaneous adipose tissue and the intra-abdominal compartment. Diabetologia. 2008;51(5):900-902.

16. Tran TT, Yamamoto Y, Gesta S, Kahn CR. Beneficial effects of subcutaneous fat transplantation on metabolism. Cell Metab. 2008;7(5):410-420.

17. Gesta $S$, et al. Evidence for a role of developmental genes in the origin of obesity and body fat distribution. Proc Natl Acad Sci U S A. 2006; 103(17):6676-6681.

18. Enerback $S$. The origins of brown adipose tissue. N Engl J Med. 2009;360(19):2021-2023.

19. Petrovic N, Walden TB, Shabalina IG, Timmons JA, Cannon B, Nedergaard J. Chronic peroxisome proliferator-activated receptor gamma (PPARgamma) activation of epididymally derived white adipocyte cultures reveals a population of thermogenically competent, UCP1-containing adipocytes molecularly distinct from classic brown adipocytes. J Biol Chem. 2010;285(10):7153-7164.

20. Ishibashi J, Seale P. Beige Can Be Slimming. Science. 2010;328(5982):1113-1114.

21. Cousin B, et al. Occurrence of brown adipocytes in rat white adipose tissue: molecular and morphological characterization. J Cell Sci. 1992;103(pt 4):931-942.

22. Ghorbani M, Himms-Hagen J. Appearance of brown adipocytes in white adipose tissue during CL 316,243-induced reversal of obesity and diabetes in Zucker fa/fa rats. Int J Obes Relat Metab Disord. 1997;21(6):465-475.

23. Guerra C, Koza RA, Yamashita H, Walsh K, Kozak LP. Emergence of brown adipocytes in white fat in mice is under genetic control. Effects on body weight and adiposity. J Clin Invest. 1998;102(2):412-420.

24. Himms-Hagen J, Melnyk A, Zingaretti MC, Ceresi E, Barbatelli G, Cinti S. Multilocular fat cells in
WAT of CL-316243-treated rats derive directly from white adipocytes. Am J Physiol Cell Physiol. 2000; 279(3):C670-C681.

25. Huttunen P, Hirvonen J, Kinnula V. The occurrence of brown adipose tissue in outdoor workers. Eur J Appl Physiol Occup Physiol. 1981;46(4):339-345.

26. Xue B, Coulter A, Rim JS, Koza RA, Kozak LP. Transcriptional synergy and the regulation of Ucp1 during brown adipocyte induction in white fat depots. Mol Cell Biol. 2005;25(18):8311-8322.

27. Barbatelli G, et al. The emergence of cold-induced brown adipocytes in mouse white fat depots is predominantly determined by white to brown adipocyte transdifferentiation. Am J Physiol Endocrinol Metab. 2010;298(6):E1244-E1253.

28. Collins S, Daniel KW, Petro AE, Surwit RS. Strainspecific response to beta 3 -adrenergic receptor agonist treatment of diet-induced obesity in mice. Endocrinology. 1997;138(1):405-413.

29. Cousin B, Casteilla L, Dani C, Muzzin P, Revelli JP, Penicaud L. Adipose tissues from various anatomical sites are characterized by different patterns of gene expression and regulation. Biochem J. 1993;292(pt 3):873-876.

30. Murano I, Zingaretti MC, Cinti S. The adipose organ of SV129 mice contains a prevalence of brown adipocytes and shows plasticity after cold exposure. Adipocytes. 2005;1:121-130.

31. Seale P, et al. PRDM16 controls a brown fat/skeletal muscle switch. Nature. 2008;454(7207):961-967.

32. Wilson-Fritch L, et al. Mitochondrial biogenesis and remodeling during adipogenesis and in response to the insulin sensitizer rosiglitazone. $\mathrm{Mol}$ Cell Biol. 2003;23(3):1085-1094.

33. Wilson-Fritch L, et al. Mitochondrial remodeling in adipose tissue associated with obesity and treatment with rosiglitazone. J Clin Invest. 2004; 114(9):1281-1289.

34. Fukui Y, Masui S, Osada S, Umesono K, Motojima $\mathrm{K}$. A new thiazolidinedione, NC-2100, which is a weak PPAR-gamma activator, exhibits potent antidiabetic effects and induces uncoupling protein 1 in white adipose tissue of KKAy obese mice. Diabetes. 2000;49(5):759-767.

35. Guan HP, Ishizuka T, Chui PC, Lehrke M, Lazar MA. Corepressors selectively control the transcrip- 
tional activity of PPARgamma in adipocytes. Genes Dev. 2005;19(4):453-461.

36. Puri V, et al. Cidea is associated with lipid droplets and insulin sensitivity in humans. Proc Natl Acad Sci U S A. 2008;105(22):7833-7838.

37. Vernochet C, et al. C/EBPalpha and the corepressors $\mathrm{CtBP} 1$ and $\mathrm{CtBP} 2$ regulate repression of select visceral white adipose genes during induction of the brown phenotype in white adipocytes by peroxisome proliferator-activated receptor gamma agonists. Mol Cell Biol. 2009;29(17):4714-4728.

38. Cederberg A, Gronning LM, Ahren B, Tasken K, Carlsson P, Enerback S. FOXC2 is a winged helix gene that counteracts obesity, hypertriglyceridemia, and diet-induced insulin resistance. Cell. 2001;106(5):563-573.

39. Dahle MK, et al. Mechanisms of FOXC2- and FOXD1-mediated regulation of the RI alpha subunit of cAMP-dependent protein kinase include release of transcriptional repression and activation by protein kinase B alpha and cAMP. J Biol Chem. 2002;277(25):22902-22908.

40. Vegiopoulos A, et al. Cyclooxygenase-2 Controls Energy Homeostasis in Mice by de Novo Recruitment of Brown Adipocytes. Science. 2010 328(5982):1158-1161.

41. Christian M, Kiskinis E, Debevec D, Leonardsson G, White R, Parker MG. RIP140-targeted repression of gene expression in adipocytes. Mol Cell Biol. 2005;25(21):9383-9391.

42. Hansen JB, et al. Retinoblastoma protein functions as a molecular switch determining white versus brown adipocyte differentiation. Proc Natl Acad Sci US A. 2004;101(12):4112-4117.

43. Scime A, et al. Rb and p107 regulate preadipocyte differentiation into white versus brown fat through repression of PGC-1alpha. Cell Metab. 2005; 2(5):283-295.

44. Tsukiyama-Kohara K, et al. Adipose tissue reduction in mice lacking the translational inhibitor 4E-BP1. Nat Med. 2001;7(10):1128-1132.

45. Wang $H$, et al. Liver $X$ receptor alpha is a transcriptional repressor of the uncoupling protein 1 gene and the brown fat phenotype. Mol Cell Biol. 2008;28(7):2187-2200.

46. Powelka AM, et al. Suppression of oxidative metabolism and mitochondrial biogenesis by the transcriptional corepressor RIP140 in mouse adipocytes. J Clin Invest. 2006;116(1):125-136.

47. Romanatto $T$, et al. Deletion of tumor necrosis factor-alpha-receptor 1 (TNFR1) protects against dietinduced obesity by means of increased thermogenesis. J Biol Chem. 2009;284(52):36213-36222.

48. Singh R, et al. Autophagy regulates adipose mass and differentiation in mice. J Clin Invest. 2009; 119(11):3329-3339.

49. Chiang $\mathrm{SH}$, et al. The protein kinase IKKepsilon regulates energy balance in obese mice. Cell. 2009; 138(5):961-975.

50. Leonardsson G, et al. Nuclear receptor corepressor RIP140 regulates fat accumulation. Proc Natl Acad SciUS A. 2004;101(22):8437-8442.

51. Pan D, Fujimoto M, Lopes A, Wang YX. Twist- 1 is a PPARdelta-inducible, negative-feedback regulator of PGC-1alpha in brown fat metabolism. Cell. 2009; 137(1):73-86.

52. Toh SY, et al. Up-regulation of mitochondrial activity and acquirement of brown adipose tissue-like property in the white adipose tissue of fsp 27 deficient mice. PLoS One. 2008;3(8):e2890.

53. Seale P, et al. Transcriptional control of brown fat determination by PRDM16. Cell Metab. 2007; 6(1):38-54.

54. Kajimura S, et al. Regulation of the brown and white fat gene programs through a PRDM16/ CtBP transcriptional complex. Genes Dev. 2008; 22(10):1397-1409.

55. Kajimura $S$, et al. Initiation of myoblast to brown fat switch by a PRDM16-C/EBP-beta transcriptional complex. Nature. 2009;460(7259):1154-1158.

56. Christian M, Parker MG. The engineering of brown fat. J Mol Cell Biol. 2010;2(1):23-25.

57. Steppan CM, et al. The hormone resistin links obesity to diabetes. Nature. 2001;409(6818):307-312.

58. Butler AA, Kozak LP. A recurring problem with the analysis of energy expenditure in genetic models expressing lean and obese phenotypes. Diabetes. 2010;59(2):323-329.

59. Ravussin E, Lillioja S, Anderson TE, Christin L, Bogardus C. Determinants of 24-hour energy expenditure in man. Methods and results using a respiratory chamber. J Clin Invest. 1986;78(6):1568-1578.

60. Bjork BC, Turbe-Doan A, Prysak M, Herron BJ, Beier DR. Prdm 16 is required for normal palatogenesis in mice. Hum Mol Genet. 2010;19(5):774-789.

61. Murano I, Barbatelli G, Giordano A, Cinti S. Noradrenergic parenchymal nerve fiber branching after cold acclimatisation correlates with brown adipocyte density in mouse adipose organ. J Anat. 2009;214(1):171-178.

62. Rappaport EB, Young JB, Landsberg L. Initiation, duration and dissipation of diet-induced changes in sympathetic nervous system activity in the rat. Metabolism. 1982;31(2):143-146.

63. Rowe JW, Young JB, Minaker KL, Stevens AL, Pallotta J, Landsberg L. Effect of insulin and glucose infusions on sympathetic nervous system activity in normal man. Diabetes. 1981;30(3):219-225.

64. Schwartz JH, Young JB, Landsberg L. Effect of dietary fat on sympathetic nervous system activity in the rat. J Clin Invest. 1983;72(1):361-370.

65 . Young JB, Saville E, Rothwell NJ, Stock MJ, Landsberg L. Effect of diet and cold exposure on norepinephrine turnover in brown adipose tissue of the rat. J Clin Invest. 1982;69(5):1061-1071.

66. Cypess AM, et al. Identification and importance of brown adipose tissue in adult humans. $N$ Engl J Med. 2009;360(15):1509-1517.

67. Saito M, et al. High incidence of metabolically active brown adipose tissue in healthy adult humans: effects of cold exposure and adiposity. Diabetes. 2009;58(7):1526-1531.

68. van Marken Lichtenbelt WD, et al. Cold-activated brown adipose tissue in healthy men. $N$ Engl J Med. 2009;360(15):1500-1508

69. Virtanen KA, et al. Functional brown adipose tissue in healthy adults. $N$ Engl J Med. 2009; 360(15):1518-1525.

70. Timmons JA, Pedersen BK. The importance of brown adipose tissue. NEnglJ Med. 2009;361(4):415-416.

71. Rodbell M. Metabolism of isolated fat cells. I. Effects of hormones on glucose metabolism and lipolysis. J Biol Chem. 1964;239:375-380.

72. Soukas A, Socci ND, Saatkamp BD, Novelli S, Friedman JM. Distinct transcriptional profiles of adipogenesis in vivo and in vitro. J Biol Chem. 2001;276(36):34167-34174.

73. Giordano A, et al. Regional-dependent increase of sympathetic innervation in rat white adipose tissue during prolonged fasting. J Histochem Cytochem. 2005;53(6):679-687. 